\title{
Partnering to advance early detection and prevention efforts for pancreatic cancer: the Florida Pancreas Collaborative
}
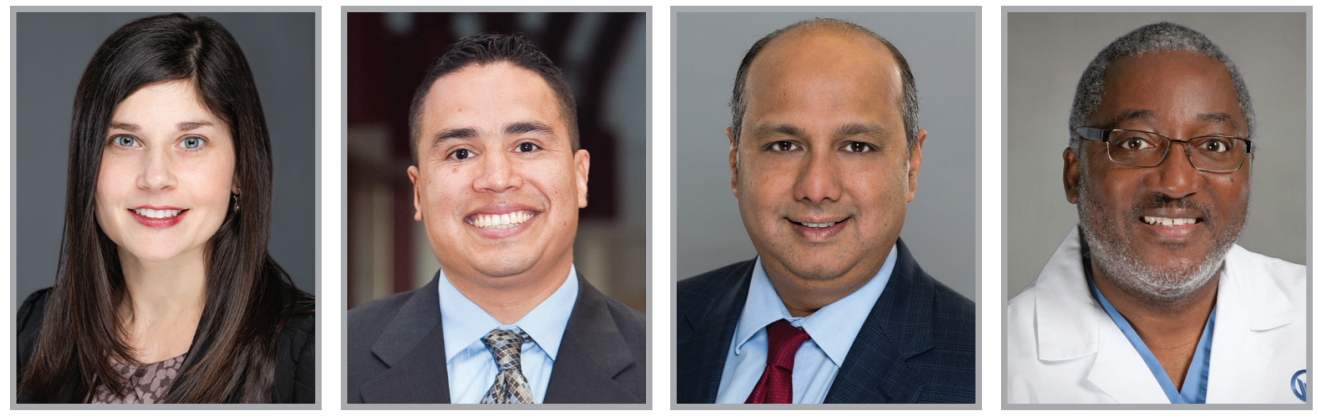

Jennifer B Permuth ${ }^{* 1,2}$, Jose Trevino ${ }^{3}$, Nipun Merchant ${ }^{4} \&$ Mokenge Malafa $^{2}$; on behalf of the Florida Pancreas Collaborative

First draft submitted: 22 January 2016; Accepted for publication: 26 January 2016; Published online: 10 February 2016

Team science as a necessity for making advancements in pancreatic cancer research

"Alone we can do so little; together we can do so much." This quote by Helen Keller embodies the overarching goal of transdisciplinary team science, which is to bring together investigators, community partners and translational collaborators from various disciplines and fields to integrate concepts, theories, methods and approaches from a breadth of expertise to solve realworld clinical problems [1]. Team science is desperately needed to make advances in the battle against pancreatic cancer, the fourth leading cause of cancer deaths in the USA and the deadliest of the top cancer killers, with a 5-year relative survival rate of only $7 \%[2]$. Based on changing demographics and incidence and death rates, pancreatic cancer is projected to surpass breast, prostate and colorectal cancer and become the second leading cause of cancer deaths by 2020 [3]. Thus, it is critical that researchers and funding agencies invest in transdisciplinary pancreatic cancer research efforts now.

\section{Focusing on early detection} \& prevention by studying commonly detected pancreatic cancer precursors

Approximately $85 \%$ of patients with pancreatic cancer present with advanced disease because of the lack of accurate methods to detect the disease at an early, operable stage [2]. The discovery of early detection and prevention strategies may

\section{KEYWORDS}

- early detection - multi-institutional collaborations $\bullet$ pancreatic cancer

“...the overarching goal of transdisciplinary team science ... is to bring together investigators, community partners and translational collaborators from various disciplines and fields to integrate concepts, theories, methods and approaches from a breadth of expertise to solve real-world clinical problems.” 
“...the Florida Academic Cancer Center Alliance was created ... to discover breakthroughs that provide greater access to cancer specialists, research, and clinical trials that result in more effective therapies and improved patient outcomes." hold the greatest promise for reducing pancreatic cancer burden, and our team and others have focused efforts on the detection and treatment of pancreatic cancer precursor lesions. Coinciding with the rise in pancreatic cancer incidence and mortality has been an increase in the radiologic detection of cystic lesions of the pancreas, including intraductal papillary mucinous neoplasms (IPMNs). IPMNs are the most common radiologically detected pancreatic cancer precursors, accounting for $40 \%$ of the approximately 150,000 asymptomatic pancreatic cysts detected incidentally in approximately one to two CT or 14-20 MRI scans per 100 cases imaged [4,5]. To date, the only way to treat these cysts and examine severity (which ranges from adenomas [low-grade] and borderline [moderate-grade] to carcinoma in situ [high-grade] and invasive [carcinoma]) is through surgical resection and pathological evaluation. However, pancreatic resection is associated with significant risks of morbidity (including long-term diabetes) and even mortality [6]. Consensus guidelines for IPMNs [7] suggest that patients with 'high-risk stigmata' (jaundice, main pancreatic duct dilation $\geq 10 \mathrm{~mm}$, or an enhanced solid component within the cyst) should undergo resection, whereas those with 'worrisome features' (acute pancreatitis, main pancreatic duct dilation 5-9 $\mathrm{mm}$, cyst size $\geq 3 \mathrm{~cm}$, thickened enhanced cyst walls, nonenhanced mural nodules) should undergo surveillance with endoscopic ultrasonography. However, recent studies $[8,9]$ have concluded that the guidelines are suboptimal in predicting pancreatic cancer risk and in maintaining a balance between under- and over-treatment. Thus, novel classes of biomarkers and imaging features and techniques are needed to predict IPMN pathology so that intervention can occur during the precancerous phase in high-risk patients before the development of malignancy.

\section{Rationale for Florida's academic cancer centers to partner in transdisciplinary pancreatic cancer research}

Florida ranks second in the number of pancreatic cancer deaths, with approximately 3000 Floridians losing their lives to this disease annually [2]. Furthermore, data from the Florida Cancer Data System (FCDS) [10] reveal that high age-adjusted pancreatic cancer incidence rates are in counties located in the primary catchment areas for the three major academic cancer centers based in Florida: Moffitt Cancer Center
(MCC; FL, USA), Sylvester Comprehensive Cancer Center at the University of Miami (UM; FL, USA) and the University of Florida Health Sciences Center (UF; FL, USA). A recent query of FCDS data [10] revealed $<20$ IPMN cases diagnosed in Florida from 2003 to 2013, a gross underestimation given that each of our centers (MCC, UF, UM) perform resections for approximately 20-25 IPMN cases per year, with all centers monitoring a number of patients suspected to be at lower risk. This underestimation is consistent with published reports in other states [11,12] and the fact that low- and moderategrade IPMNs currently represent nonreportable conditions in Florida. Based on our observations of this underestimation, the FCDS is in discussion with the Florida Department of Health to attempt to change the state reporting requirements (personal communication, FCDS liaison).

Given the growing incidental detection of IPMNs and the major clinical challenges that they pose, a transdisciplinary group of researchers led by a molecular epidemiologist (JB Permuth) and pancreatic surgeons (J Trevino, N Merchant and M Malafa) at our three centers recognized that an important opportunity existed to establish a state-wide infrastructure to prospectively identify, characterize and monitor incident IPMN cases and evaluate putative risk factors for and biomarkers of pancreatic cancer development and progression.

\section{The establishment of the Florida Pancreas} Collaborative

In 2014, the Florida Academic Cancer Center Alliance [13] was created by the State of Florida and leaders at MCC, UM and UF to discover breakthroughs that provide greater access to cancer specialists, research, and clinical trials that result in more effective therapies and improved patient outcomes. In autumn 2015, our team secured funds from the Florida Academic Cancer Center Alliance to establish the Florida Pancreas Collaborative, the first state-wide multi-center collaboration that we know of dedicated to conducting research for patients with IPMNs with the intent of promoting the early detection and prevention of pancreatic malignancy.

The Florida Pancreas Collaborative is working to overcome deficiencies of existing pancreatic cancer registries and biorepositories [14-16] with several enhancements. We will target a greater breadth of pancreas disease cases. By recruiting a consecutive series of males and 
females who are $\geq 18$ years old presenting to clinics at MCC, UF or UM with a high clinical suspicion for (or diagnosis of) a pancreatic lesion or pancreatitis based on symptoms, crosssectional imaging or blood work, we will be able to recruit late- and early-stage pancreatic cancer cases, IPMNs, a less common pancreatic cancer precursor called mucinous cystic neoplasms [17], and benign conditions such as chronic pancreatitis [18] and nonmucinous pancreatic cysts such as serous cystadenomas and pseudocysts [19]. This will enable discovery of biomarkers that can distinguish between malignant, premalignant and benign conditions [17-20]. We will prospectively acquire, process and store a variety of biospecimens (including blood and cyst fluid) rather than relying solely on tumor tissue, which may have suboptimal [14] or limited [2] use. These samples will be collected using standard operating procedures on a longitudinal basis (at baseline/preoperatively and postoperatively or during surveillance), typically occurring in conjunction with standard of care procedures. We will recruit healthy controls without a personal history of pancreatic disease as a comparison group for etiologic investigations and biomarker development. In particular, those at high genetic risk for pancreatic cancer [21] are excellent candidates for chemoprevention and surveillance studies.

For patients with pancreatic diseases, we are using questionnaires to collect common data elements related to demographics, presenting systems, past medical history, past surgical history, family history of cancer, current and past medication use, and information on a uniform set of known and suspected cancer risk or protective factors. Surgical details, pathology results, lab values, treatment and follow-up information are also being obtained from electronic medical records and other source systems. The questionnaires also serve as the main data source for healthy controls. Abstracted data are loaded into a centralized virtual repository using the software solution, Research Electronic Data Capture (REDCap) [22], maintained by the team. In the future, we will be designing a follow-up questionnaire that will be administered annually. We have already developed a flyer and informational brochure to aid in recruitment efforts at each of our institutions.

During this 1-year pilot project, we plan to prospectively recruit a cohort of approximately 100 Floridians newly diagnosed with IPMNs and other pancreatic conditions (and a similar number of healthy controls) and generate a comprehensive data and specimen repository that complements existing single institution protocols; use the multi-institutional repository to validate findings generated by our MCC team [23], which suggest that a plasma-based miRNA classifier may serve as a promising diagnostic adjunct to distinguish 'malignant' IPMNs requiring surgically resection from 'benign' IPMNs that can be monitored; and generate preliminary data to support the potential for other novel classes of candidate molecular markers and imaging features to differentiate between malignant and benign IPMNs. Since the time of our study's inception, we have recruited clinical researchers from other disciplines (radiology, gastroenterology, endoscopy, oncology, pathology, immunology, genetics) along with biostatisticians, bioinformaticists, basic scientists and patient advocates to help accomplish these and future objectives.

\section{Final perspective}

In summary, transdisciplinary team science is essential to support research for the development and testing of new molecular and imaging biomarkers to identify patients at high risk for pancreatic cancer because of the presence of precursor lesions, genetic factors, or other risk factors and co-morbidities such as pancreatitis and diabetes. The Florida Pancreas Collaborative represents researchers, doctors, patients and families united in efforts to prevent, detect and treat pancreatic cancer. We also seek to partner with other cancer centers and stakeholders in Florida and beyond to make advances by conducting large-scale multicenter longitudinal studies. This process will take time and investment but will undoubtedly improve medical decisions, support personalized care and ultimately save lives, consistent with the mission at our respective cancer centers and our collective mission to contribute to the prevention and cure of cancer.

\section{Acknowledgements}

The authors thank $R$ Hamilton, ELS, for her editorial assistance.

\section{Financial \& competing interests disclosure}

The authors wish to acknowledge that this partnership is supported by the State of Florida and the Florida Academic Cancer Center Alliance. The authors also wish to acknowledge Institutional Research Grant number
“The Florida Pancreas Collaborative represents researchers, doctors, patients and families united in efforts to prevent, detect and treat pancreatic cancer.” 
93-032-16 from the American Cancer Society (JB Permuth) and the Collaborative Data Services, Tissue, Molecular Genomics, Biostatistics and Cancer Informatics Core Facilities at the H Lee Moffitt Cancer Center \& Research Institute, an NCI designated Comprehensive Cancer Center (P30-CA076292). The authors have no other relevant affliations or financial involvement with any organization or entity with a financial interest in or financial conflict with the subject matter or materials discussed in the manuscript apart from those disclosed.

No writing assistance was utilized in the production of this manuscript.

\section{References}

1 Stokols D, Hall KL, Vogel AL.

Transdisciplinary public health: definitions, core characteristics, and strategies for success. In: Transdisciplinary Public Health: Research, Methods, and Practice. Haire-Joshu $\mathrm{D}, \mathrm{McBride} \mathrm{T}$ (Eds). Jossey-Bass, CA, USA, 3-30 (2013).

2 American Cancer Society. Cancer Facts and Figures 2015. American Cancer Society, Atlanta, GA, USA (2015). www.cancer.org

3 Rahib L, Smith BD, Aizenberg R, Rosenzweig $A B$, Fleshman JM, Matrisian LM. Projecting cancer incidence and deaths to 2030: the unexpected burden of thyroid, liver, and pancreas cancers in the United States. Cancer Res. 74(11), 2913-2921 (2014).

4 Megibow AJ, Baker ME, Gore RM, Taylor A. The incidental pancreatic cyst. Radiol. Clin. North Am. 49(2), 349-359 (2011).

5 Matthaei H, Schulick RD, Hruban RH, Maitra A. Cystic precursors to invasive pancreatic cancer. Nat. Rev. Gastroenterol. Hepatol. 8(3), 141-150 (2011).

6 Hines OJ, Reber HA. Pancreatic surgery. Curr. Opin. Gastroenterol. 24(5), 603-611 (2008).

7 Tanaka M, Fernandez-del Castillo C, Adsay $\mathrm{V}$ et al. International consensus guidelines 2012 for the management of IPMN and MCN of the pancreas. Pancreatology 12(3), 183-197 (2012).

8 Roch AM, Ceppa EP, DeWitt JM et al. International Consensus Guidelines parameters for the prediction of malignancy in intraductal papillary mucinous neoplasm are not properly weighted and are not cumulative. HPB (Oxford) 16(10), 929-935 (2014).

9 Sahora K, Mino-Kenudson M, Brugge W et al. Branch duct intraductal papillary mucinous neoplasms: does cyst size change the tip of the scale? A critical analysis of the revised international consensus guidelines in a large single-institutional series. Ann. Surg. 258(3), 466-475 (2013).

10 Florida Cancer Data System. http://fcds.med.miami.edu

11 Le H, Ziogas A, Rhee JM, Lee JG, Lipkin SM, Zell JA. A population-based, descriptive analysis of malignant intraductal papillary mucinous neoplasms of the pancreas. Cancer Epidemiol. Biomarkers Prev. 17(10), 2737-2741 (2008).

12 Reid-Lombardo KM, St Sauver J, Li Z, Ahrens WA, Unni KK, Que FG. Incidence, prevalence, and management of intraductal papillary mucinous neoplasm in Olmsted County, Minnesota, 1984-2005: a population study. Pancreas 37(2), 139-144 (2008).

13 The Florida Academic Cancer Center Alliance. www.floridacanceralliance.com

14 Hwang RF, Wang H, Lara A et al. Development of an integrated biospecimen bank and multidisciplinary clinical database for pancreatic cancer. Ann. Surg. Oncol. 15(5), 1356-1366 (2008).

15 Demeure MJ, Sielaff T, Koep L et al. Multi-institutional tumor banking: lessons learned from a pancreatic cancer biospecimen repository. Pancreas 39 (7), 949-954 (2010).
16 Amin W, Singh H, Dzubinski LA, Schoen RE, Parwani AV. Design and utilization of the colorectal and pancreatic neoplasm virtual biorepository: an early detection research network initiative. J. Pathol. Inform. 1, 22 (2010).

17 Hruban RH, Maitra A, Kern SE, Goggins M. Precursors to pancreatic cancer. Gastroenterol. Clin. North Am. 36(4), 831-849 (2007).

18 Cecconi D, Palmieri M, Donadelli M. Proteomics in pancreatic cancer research. Proteomics 11(4), 816-828 (2011).

19 Khalid A, Brugge W. ACG practice guidelines for the diagnosis and management of neoplastic pancreatic cysts. Am. J. Gastroenterol. 102(10), 2339-2349 (2007).

20 Goggins M. Identifying molecular markers for the early detection of pancreatic neoplasia. Semin. Oncol. 34(4), 303-310 (2007).

21 Canto MI, Goggins M, Hruban RH et al. Screening for early pancreatic neoplasia in high-risk individuals: a prospective controlled study. Clin. Gastroenterol. Hepatol. 4(6), 766-781; quiz 665 (2006).

22 Harris PA, Taylor R, Thielke R, Payne J, Gonzalez N, Conde JG. Research electronic data capture (REDCap) - a metadata-driven methodology and workflow process for providing translational research informatics support. J. Biomed. Inform. 42(2), 377-381 (2009).

23 Permuth-Wey J, Chen DT, Fulp WJ et al. Plasma microRNAs as novel biomarkers for patients with intraductal papillary mucinous neoplasms of the pancreas. Cancer Prev. Res. (Phila.) 8(9), 826-834 (2015). 